\title{
Advanced Receiver and MIMO Schemes for LTE Communications System
}

\author{
Simone Morosi, Enrico Del Re, Luca Vettori \\ Department of Electronics and Telecommunications \\ University of Florence, Italy
}

\begin{abstract}
Within the 3rd Generation partnership project (3Gpp) the LTE standard is currently being defined aiming at providing rates up to $300 \mathrm{Mb} / \mathrm{s}$ in downlink. In this paper, main alternative strategies which have been proposed for the Downlink air interface are compared by taking into account different modulation formats and transmit diversity schemes. Moreover, we also consider the modulation and coding tecniques which have been previously used in the WiMAX system.
\end{abstract}

\section{INTRODUCTION}

The Universal Mobile Telecommunications System (UMTS) is foreseen to provide high speed packet access for both the downlink (up to $14 \mathrm{Mbps}$, thanks to the HSPDA system) and the uplink (up to $5.76 \mathrm{Mbps}$, with the HSUPA). Nonetheless, with the definition of packet-based wireless broadband systems such as the WiMAX IEEE 802.16e, it has been largely understood that a "Long Term Evolution" (LTE) of UMTS systems was needed to remain competitive on the long term.

Therefore the 3Gpp, in December 2004, has initiated a specific activity on the long term evolution of UTRAN for the market introduction of LTE around 2010, by affording a functional freeze (as part of Release 8) in December 2008. The principal goals and requirements for LTE [1] are the following:

- Downlink peak data rates up to $300 \mathrm{Mbps}$ and uplink peak data rates up to $80 \mathrm{Mbps}$;

- Multi-antenna configurations;

- Significantly reduced control-plane latency;

- High spectral efficiency both in downlink and in uplink;

- Increase of the User Throughput, even at the cell edge;

- Scalable bandwidth up to $20 \mathrm{MHz}$

- Support for packet switched (PS) domain only;

- Full user mobility supported and connections maintained with mobile speeds even up to $350 \mathrm{kmph}$;

- Reduced cost for operator and end user.

In the Up-Link, Single-Carrier Frequency Division Multiple Access (SC-FDMA) has been selected to efficiently meet E-UTRA performance requirements [1]. The choose of SCFDMA in up-link was considered due to its intrisec relative low Peak-to-Average Power Ratio (PAPR) [1], which is a key requirement on mobile terminal for conserving battery life or extending range.

On the other hand, in the Down-Link, Orthogonal Frequency Division Multiple Access (OFDMA) has been selected to afford the requirements: as is known, the OFDM system allows to effectively exploit frequency selectivity of the multi-path channel with low-complexity receivers.

In this paper, main different alternatives which have been proposed for the downlink air interface are compared by taking into account different modulation formats and transmit diversity schemes. Moreover, we also consider the modulation and coding tecniques which have been previously used in the WiMAX [6] system.

This paper is organized as follows. In Section II the DownLink System model chosen for our simulations is presented . In the Sections III and IV multi antenna and coding technique are introduced and investigated. In the Section $\mathrm{V}$ we present the results of our simulations with some concluding remarks in Section VI.

\section{DOWN-LINK System ModeL}

In the down-link communications, LTE system is based on the OFDM modulation. This solution has been chosen due to the moderate complexity of the receivers in case of large bandwidth, for the robustness against multipath fading and the high spectral efficiency. Moreover, on the the network side the transmission PAR was not considered as a key problem.

The OFDM technique [8] differs from traditional FDM: in this system multiple carriers (called subcarriers) carry the information stream, sub-carriers are orthogonal to each other, and a guard time may be added to each symbol to combat the channel delay spread. In practice, the OFDM signal can be generated using IFFT digital signal processing. Therefore, it is easy to understand that, from a parallel stream of $N$ sources of data, each one modulated with QAM useful symbol period $T_{u}$, a waveform composed of $\mathrm{N}$ orthogonal subcarriers is obtained.

Fig.1 represents the mapping from a serial stream of QAM symbols to $\mathrm{N}$ parallel streams, used as frequency domain bins for the IFFT. The N-point time domain blocks obtained from the IFFT are then serialized to create a time domain signal.

A guard interval may be added prior to each useful OFDM symbol. This guard time is introduced to minimize the interOFDM-symbol-interference caused by time-dispersive channels. The guard interval duration $T_{g}$ (which corresponds to $N_{p}$ prefix samples) must be long enough to cover the most of the delay-spread energy of a radio channel impulse response. In the guard interval prior a prefix using the last block of $N_{p}$ samples from the symbol itself may be inserted: this prefix is usually called cyclic prefix. 


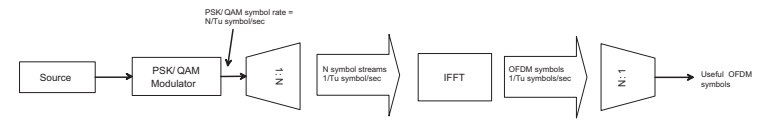

Figure 1. OFDM Signal Generation Chain

The access technology of the LTE, which is called ScalableOFDMA, can be achieved by the OFDM by introducing frequency selective scheduling and the number of subcarriers that scales with bandwidth: in fact, through the information obtained by downlink quality channel, the scheduler determines how many and which downlink physical resource blocks allocate to the users and selects an appropriate data-rate for each physical resource block by varying the output power level, the channel coding rate and/or the modulation scheme (up to 64QAM). Note that the downlink physical resource blocks are identified by the number of subcarriers in the time and frequency domains.

As mentioned the bandwith scales from $1.4 \mathrm{MHz}$ to 20 $\mathrm{MHz}$ : as a result, also the sampling frequency (from $1.92 \mathrm{MHz}$ to $30.72 \mathrm{MHz}$ ), the size of internal FFT (from 128 to 2048) and the number of occupied subcarriers (from 72 to 1200) varies accordingly. On the contrary, the subcarrier spacing, and, thus, the OFDM symbol period, remains the same, i.e., $\Delta f=15 k H z^{1}$.

\section{Space Time Coding Schemes}

In the following we analize how to exploit the diversity gain by using the Space-Time Coding (STC) scheme in the LTE system. Between the MIMO techniques ${ }^{2}$ the (STC) affords robustness against noise, interference and multipath fading. It was introduced by Alamouti in his pionerestic paper[7] and it is largely used because combines diversity with receiver structure with low complexity; it has been recently generalized to the OFDM systems, so defining the MIMO-OFDM technique, and more specifically, the Space-Time Block Code (STBC) and Space-Frequency Block Code (SFBC) which are described in the following.

First, we define the notation for the OFDM symbols in the frequency domain. With $S^{(n)}=\left[S_{0}^{(n)} S_{1}^{(n)} \ldots S_{N-1}^{(n)}\right]^{T}$ we indicate the OFDM symbol consisting of $N$ sub-carriers, where $S_{k}^{(n)}$ can be viewed as the data symbol to be transmitted on the $k$-th subcarrier during the block instant $n$.

In order to apply the Space-Time Block Coding (STBC) technique which has been defined in [9], we must consider the transmission in two consecutive OFDM symbol periods. Without Space-Time Coding we transmit the OFDM symbol $S^{(a)}$ in the first symbol period and $S^{(b)}$ in the second symbol period. With the Alamouti Coding in time domain, in the first OFDM symbol period, the data symbol $S_{l}^{(a)}$ is transmitted on the $l$-th subcarrier over the first transmitting antenna and the

${ }^{1}$ Hence, the OFDM symbol period is $T_{u}=\frac{1}{\Delta f}=66.67 \mu \mathrm{s}$

${ }^{2}$ With MIMO (Multiple Input Multiple Output)system we mean the presence of more than one antenna both at the transmitter and the receiver side. data symbol $S_{l}^{(b)}$ is transmitted on the $l$-th subcarrier over the second transmitting antenna.

On the contrary, in the second OFDM symbol period, the data symbol $-S_{l}^{*(b)}$ is transmitted on the $l$-th subcarrier over the first transmitting antenna and the data symbol $+S_{l}^{*(a)}$ is transmitted on the $l$-th subcarrier over the second transmitting antenna. Therefore, we obtainthe Alamouti Coding for each sub-carriers of OFDM symbol.

The Space-Frequency Block Coding (SFBC) [10] technique performs the Alamouti coding over two consecutive subcarriers of the OFDM symbol: psrticularly, the OFDM symbol $S^{(n)}$ is coded into two vectors $S_{1}^{(n)}$ and $S_{2}^{(n)}$ by the space-frequency encoder block as

$$
\begin{gathered}
S_{1}^{(n)}=\left[\begin{array}{llll}
S_{0}^{(n)} & -S_{1}^{*(n)} \cdots & S_{N-2}^{(n)} & -S_{N-1}^{*(n)}
\end{array}\right]^{T} \\
S_{2}^{(n)}=\left[\begin{array}{llll}
S_{1}^{(n)} & +S_{0}^{*(n)} \cdots & S_{N-1}^{(n)}+S_{N-2}^{*(n)}
\end{array}\right]^{T} .
\end{gathered}
$$

Therefore, in the $n$-th interval, we transmit $S_{1}^{(n)}$ from the first transmitting antenna and $S_{2}^{(n)}$ simultaneously from the second transmitting antenna.Hence, the operations of space-frequency coding and decoding can be better described in terms of even and odd component vectors. Let $S_{e}^{(n)}$ and $S_{o}^{(n)}$ be two vectors (with length $\frac{N}{2}$ ) that represent the even and odd component of the original vector $S^{(n)}$.

$$
\begin{gathered}
S_{e}^{(n)}=\left[\begin{array}{llll}
S_{0}^{(n)} & S_{2}^{(n)} \cdots & S_{N-4}^{(n)} & S_{N-2}^{(n)}
\end{array}\right]^{T} \\
S_{o}^{(n)}=\left[\begin{array}{llll}
S_{1}^{(n)} & S_{3}^{(n)} \cdots & S_{N-3}^{(n)} S_{N-1}^{(n)}
\end{array}\right]^{T} .
\end{gathered}
$$

Accordingly, the $S_{1, e}^{(n)}, S_{1, o}^{(n)}, S_{2, e}^{(n)}$ and $S_{2, o}^{(n)}$ represent the even and odd component vectors of $S_{1}^{(n)}$ and $S_{2}^{(n)}$ (OFDM symbols coded from original symbol $S^{(n)}$ ).

Then, let $\Lambda_{1}^{(n)}$ and $\Lambda_{2}^{(n)}$ be two diagonal matrices whose elements are the DFTs of the respective channel impulse response, $h_{1}^{(n)}$ and $h_{2}^{(n)}$. The demodulated signal at the receiver can be derived in the frequency domain as

$$
Y^{(n)}=\Lambda_{1}^{(n)} \cdot S_{1}^{(n)}+\Lambda_{2}^{(n)} \cdot S_{2}^{(n)}+Z^{(n)} .
$$

Assuming that the channel responses are known or, at least, can be estimated accurately at the receiver, the Alamouti combining schemes can be implemented building the vector $\tilde{S}$ whose even and odd components are equal to

$$
\begin{gathered}
\tilde{S}_{e}^{(n)}=\Lambda_{1, e}^{*(n)} \cdot Y_{e}^{(n)}+\Lambda_{2, o}^{(n)} \cdot Y_{o}^{*(n)} \\
\tilde{S}_{o}^{(n)}=\Lambda_{2, e}^{*(n)} \cdot Y_{e}^{(n)}-\Lambda_{1, o}^{(n)} \cdot Y_{o}^{*(n)} .
\end{gathered}
$$

Another assumption is to consider that the complex channel gains between adjacent sub-carriers are approximately costant, i.e., $\Lambda_{1, e}^{(n)} \approx \Lambda_{1, o}^{(n)}$ and $\Lambda_{2, e}^{(n)} \approx \Lambda_{2, o}^{(n)}$; therefore, the equations (3) and (4) become

$$
\begin{aligned}
& \tilde{S}_{e}^{(n)}=\left(\left|\Lambda_{1, e}^{(n)}\right|^{2}+\left|\Lambda_{2, e}^{(n)}\right|^{2}\right) \cdot S_{e}^{(n)}+\Lambda_{1, e}^{*(n)} \cdot Z_{e}^{(n)}+\Lambda_{2, o}^{(n)} \cdot Z_{o}^{*(n)} \\
& \tilde{S}_{o}^{(n)}=\left(\left|\Lambda_{1, o}^{(n)}\right|^{2}+\left|\Lambda_{2, o}^{(n)}\right|^{2}\right) \cdot S_{o}^{(n)}+\Lambda_{2, e}^{*(n)} \cdot Z_{e}^{(n)}-\Lambda_{1, o}^{(n)} \cdot Z_{o}^{*(n)} .
\end{aligned}
$$




\section{Channel Coding Schemes}

Turbo Codes and Tail Biting Convolutional Codes have been proposed for the channel coding of Traffic Channel in LTE [3]: particularly, Turbo Coding is used with UpLink Shared Channel (UL-SCH), DownLink Shared Channel (DL$\mathrm{SCH})$, Paging Channel (PCH) and Multicast Channel (MCH) whereas Broadcasting Channel (BCH) uses Tail Biting Convolutional Coding.

Concerning the Turbo Encoder, LTE system resotrs to the scheme which has been used in the UMTS standard [3] which is based on two Recursive Systematic Convolutional (RSC) encoders connected by an interleaver: particularly, the RSC constituent encoders are 8-state whose transfer function is:

$$
G(D)=\left[1, \frac{g_{1}(D)}{g_{0}(D)}\right]
$$

with

$$
\begin{gathered}
g_{0}(D)=1+D^{2}+D^{3} \\
g_{1}(D)=1+D+D^{3}
\end{gathered}
$$

The internal interleaver is a 6144 input-bit with limited memory. Therefore, the total coderate is $1 / 3$.

Concerning the tail-biting (TB) convolutional coding the LTE scheme uses a convolutional code with constraint length $K=7$ and coding rate $1 / 3$ (as for the Turbo coding rate). The configuration of the convolutional encoder is presented in Fig. 2.

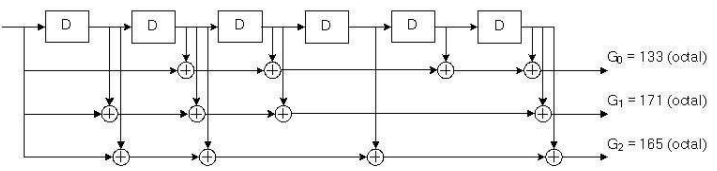

Figure 2. Tail Biting Convolutional Encoder with rate 1/3 proposed for LTE

The main disadvantage of the tail-biting technique is that the maximum likelihood decoder (still based on the Viterbi Algorithm) is far more complex than the classical convolutional decoder: as reported in literature [5], the Maximum Likelihood Decoding (MLD) of a TB code is optimum in term of performance, but not of Viterbi Trials (VT) and, as a consequence, of decoding time. In order to reduce the number of VT we have considered a modified version of the algorithm developed by Wang and Bhargava in [5].

First, we describe the original algorithm. We suppose to have a trellis $T_{N}(B, S, E)$ (that is a trellis with length $\mathrm{N}$, set of all states of trellis $\mathrm{S}$, set of starting states $\mathrm{B}$ and set of ending states E) ${ }^{3}$. In the case of the Ideal Algorithm in a VT all the set of starting states B (not only one starting state) are considered "active". So, the final survivor for each state in $E$ is the path of the largest metric from any starting state in $B$ to that state. Now we can present the Real Algorithm: we suppose to know the trellis of a FTB code $T_{N}(B, S, E)$ where

\footnotetext{
${ }^{3}$ In the case of Full Tail Biting both $\mathrm{B}$ and $\mathrm{E}$ are all the $2^{K-1}$ possible states of trellis
}

$\mathrm{B}$ e $\mathrm{E}$ are the entire set of possible state of trellis. If a path starts from an element in $\mathrm{B}$, say $b_{i}$ and ends to a state in $e_{i}$ in $\mathrm{E}$ for the same $\mathrm{i}$, we define the path as qualified from $\mathrm{B}$ to E. The same notation can be adopted for B, and E, as will be specified below.

1) Initialize the metric of the candidate 4 by $-\infty$;

2) Set $B_{t}=B$ and $E_{t}=E$, and perform Algorithm 1 through $T_{N}(B, S, E)$;

3) Determine the path $M$ with the largest metric among all survivors ending at states in E. If its metric is not larger than that of the candidate, stop. Otherwise, go to step 4;

$4)$ If there does not exist any survivor which is qualified from $B_{t}$ to $E_{t}$, go to step 6 . Otherwise (note that there may be more than one qualified survivor), find and store the qualified path, denoted as $\mathrm{C}$, with the largest metric. If its metric is larger than that of the candidate, update the candidate with $\mathrm{C}$. If the metric of $\mathrm{C}$ is equal to that of $\mathrm{M}$, stop. Otherwise, go to step 5;

5) Reduce B by deleting from it those elements $b_{i}$ 's which are starting states of qualified survivors recognized in step 4 and reduce $\mathrm{E}$ by deleting the corresponding $e_{i}$ 's from it. If B (or E) is not empty, repeat step 2. Otherwise, stop.

6) Reduce $B_{t}$ by deleting $\left\lfloor\left\|B_{t}\right\| / 2\right\rfloor$ or $\left[\left\|B_{t}\right\| / 2\right\rceil$ number of its elements $b_{i}$ s which include starting states of more survivors than those elements which are not deleted from $B_{t}$ and reduce $E_{t}$ by deleting the corresponding $e_{i}$ s from it. The resultant $\mathrm{B}$, and $\mathrm{E}$, must not be empty. Here, $\left\|B_{t}\right\|$ is the order of $B_{t}$. Perform Algorithm 1 through $T_{N}(B, S, E)$. Go to step 4 .

So the final candidate obtained in previous Algorithm is the maximum likelihood path of the FTB code.

As it is said in [5] the number of VT decreases when the signal-to-noise ratio increases, therefore the complexity of algorithm is asymptotically equal to the Viterbi algorithm for high signal-to-noise ratio. To reduce the complexity also for lower SNR we have changed the original algorithm, by eliminating the Step 5 and terminating the algorithm in the previous step. The result from this variation is the candidate whose qualified path has the largest metric instead of the path with largest metric even if it is not qualified. With this modification, even for moderate SNR, the Wang-Bhargava algorithm reduces its number of VT and decoding time.

Finally, we have considered a channel coding scheme which has not been proposed for the LTE system: in particular, we have studied the channel coding proposed in IEEE 802.16 standard (WiMAX standard), trying to adapt its characteristic to our LTE system model. Basically, the WiMAX Channel coding [6] is composed by a convolutional encoder connected to an interleaver.

- The inner structure of the convolutional encoder is reported in Fig.3. As we can see, the encoder has cod-

\footnotetext{
${ }^{4}$ On performing the algorithm once through the trellis of a FTB code, we may get a path of the largest metric having proper starting and ending states. This path is called a candidate.
} 
ing rate of $1 / 2$ and constraint length $K=7$. For our simulations we use this convolutional encoder combined with the Tail-Biting technique (using the modified WangBhargava decoding algorithm which has been previously described).

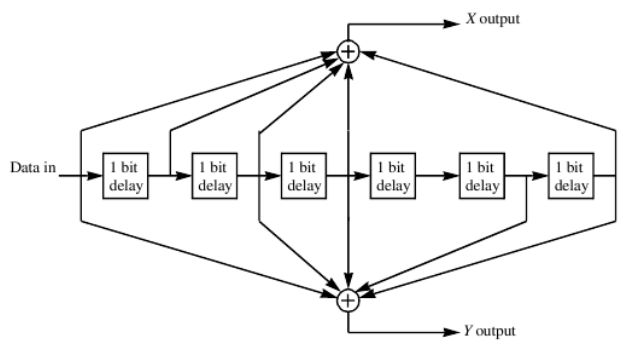

Figure 3. Inner Structure of Convolutional Encoder proposed in WiMAX standard.

- The interleaver is defined by a two step permutation. The first ensures that adjacent coded bits are mapped onto non-adjacent subcarriers. The second permutation guarantees that adjacent coded bits are mapped alternately onto less or more significant bits of the constellation, thus avoiding long runs of lowly reliable bits.

In this case we must adapt some characteristic of the emcoder to LTE system: in particular, we change the value of inner permutation to 18 , while in the WiMAX standard it is equal to 12 .

\section{Simulations Results}

In the following, we report the results of the simulations of LTE system; as in the system proposal, the parameter values have the following values:

- Bandwith = $3 \mathrm{MHz}$;

- Sampling Frequency $=3.84 \mathrm{MHz}$;

- FFT Size = 256;

- Number of occupied subcarriers $=181^{5}$;

- Number of OFDM symbols per sub-frame $=14$;

- CP lenght (Short) $=5.21 \mu s$ and $4.69 \mu s$ corresponding to $20 / 18$ samples.

Moreover, we assume the presence of only a single user, that is only one user has allocated all the 180 occupied subcarrier and consequently all the physical resource blocks. For what concerns the channel coding, for all the simulations we use the $\log -M A P$ Algorithm as decoding algorithm in the Turbo Decoder, since it permits of achieve good performance with a complexity lower than the MAP Algorithm. As we said before, we use the modified version of Wang-Bhargava Algorithm as decoding algorithm. Concerning the antenna schemes of the LTE system, we analize the SISO case (Single Input Single Output, i.e., only one transmitting antenna and only one receiving antenna) and the MIMO case: particularly, for the latter case we implement both the STBC and the SFBC schemes in the $2 \times 1$ and $2 \times 2$ cases. This means that we use

\footnotetext{
${ }^{5}$ Includes DC subcarrier which contains no data
}

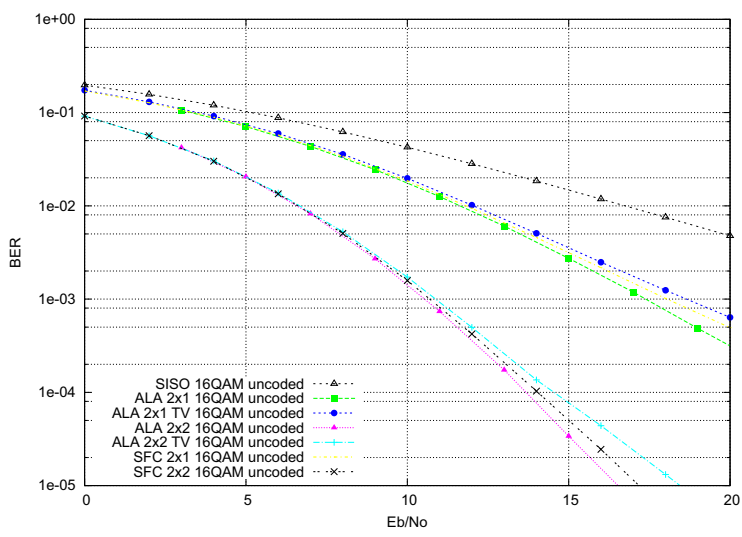

Figure 4. Uncoded BER performance for 16QAM modulation and different multi-antenna configuration.

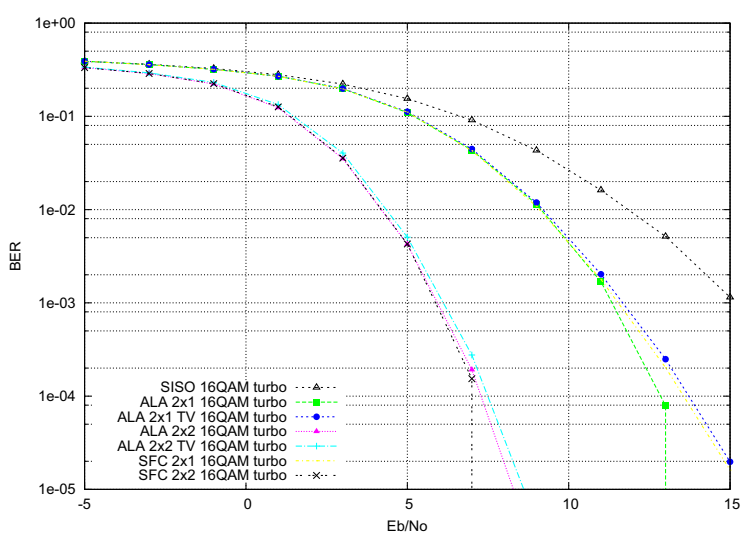

Figure 5. BER performance with turbo-codingfor 16QAM modulation and different multi-antenna configuration.

2 antennas at the transmitter and 1 or 2 antennas at the receiver. All the simulations are relative to a multipath channel with Rayleigh fading (NLOS ambient) at carrier frequency of $1850 \mathrm{MHz}$ and high user mobility. Regarding the STBC case we have considered both the case of constant fading across two consecutive OFDM symbols and of variable fading across two consecutive symbols (Time-Varying, TV). Perfect channel estimation is considered. Finally, we have used a ZF (Zero Forcing) equalization in the receivers. Fig.4 show simulation results in terms of uncoded BER as a function of the signal to noise ratio (expressed in $\mathrm{dB}$ ) for a 16QAM modulation. The performance of the Alamouti schemes (in the time domain) with constant fading channels (ALA2x1 and ALA2 2 ) achieve better performance compared to the equivalent cases with non-constant channels (ALA2x1 TV and ALA2 $22 \mathrm{TV})$. An interesting result is that the Alamouti schemes in frequency domain (SFC $2 \times 1$ and SFC $2 \times 2$ ) have similar performance compared to equivalent schemes in time domain.

The same trend is obtained with the introduction of 3GPP Release 6 Turbo Coding as we can observe from Fig.5.

In Fig.6 we report the performance in terms of Bit Error Rate (BER) in the case of QPSK modulation and SISO 


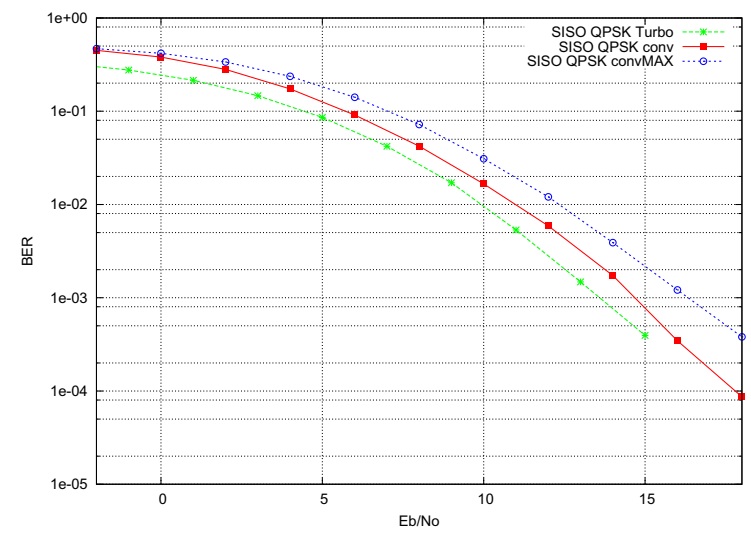

Figure 6. BER Performance for QPSK modulation and different channel coding, single-input single-output.

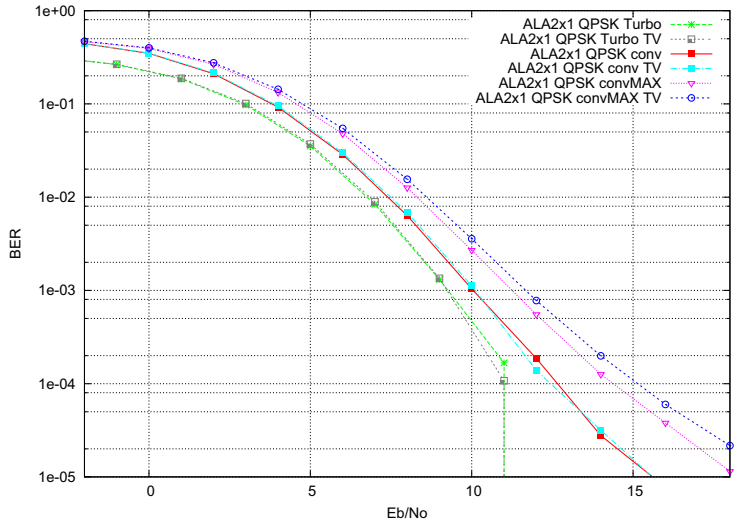

Figure 7. BER Performance for QPSK modulation and different channel coding, Alamouti configuration ( 2 trasmitting antennas and 1 receiving antenna)

configuration antennas. The Convolutional Coding proposed for the LTE, has better performance compared to the WiMAX convolutional encoder (with its own interleaver), adapted to the LTE model. As we can see Turbo Coding results are obviously better compared to the other used convolutional coding.

In Fig.7 we report the BER performance in case of QPSK modulation and Alamouti Coding (in time domain) with 2 transmitting and 1 receiving antennas: with respect to the SISO case all the schemes are characterized by better performance; this performance gain is due to the Alamouti Coding which exploits the diversity in trasmission. In the case with nonconstant fading channel across two consecutive symbols period, we can observe the same trends reported in the SISO case.

In Fig.8 we report the performance in terms of Bit Error Rate (BER) in case of QPSK modulation and Alamouti Coding (alwais in time domain) with 2 antennas both at the transmitter and receiver side, also with the case of non-constant fading channel. Also in this case we observe a remarkable improvement in BER for the presence of the second receiving antenna.

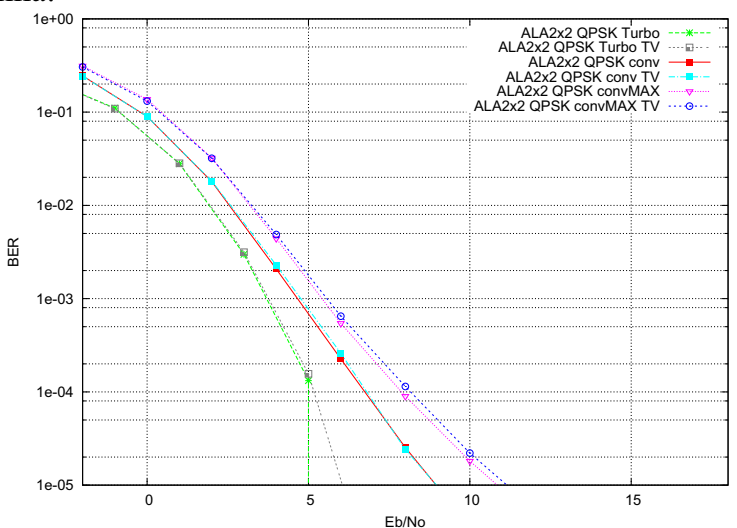

Figure 8. BER Performance for QPSK modulation and different channel coding, Alamouti configuration (2 trasmitting antennas and 2 receiving antenna)

\section{CONCLUSIONS}

In this paper, main different alternatives which have been proposed for the Down-Link air interface have been compared by taking into account different modulation formats and transmit diversity schemes. Moreover, we have also considered the modulation and coding tecniques which have been previously used in the WiMAX system. Turbo codes are characterized by better performance for all the diversity schemes and modulation formats. Nonetheless, coding schemes with significantly lower complexity, such as TB convolutional codes and WiMAX coding, shows good performance in all the case which have been considered. It is worth underline that the MIMO schemes based on SFBC show the same performance of the classical STBC systems with lower requirements in term of channel conditions.

\section{REFERENCES}

[1] A. Toskala, H. Holma, K. Pajukoski, E. Tiirola "UTRAN Long Term Evolution in 3GPP", IEEE PIMRC,2006.

[2] 3GPP "Long Term Evolution (LTE) physical layer; General description" TS 36.201 V8.2.0, 2008-12.

[3] 3GPP "E-UTRA Multiplexing and channel coding" TS 36.212 V 8.5.0, 2008-12

[4] H. Ma and J. Wolf "On Tail Biting Convolutional Codes" IEEE Transactions on Communications, Vol. 34, February 1986.

[5] Q. Wang and V. Bhargava "An Efficient Maximum Likelihood Decoding Algorithm for Generalized Tail Biting Convolutional Codes Including Quasicyclic Codes" IEEE Transactions on Communications, Vol.37, August 1989.

[6] IEEE "Air Interface for Fixed Broadband Wireless Access Systems" IEEE Std 802.16-2004, 2004.

[7] S.A. Alamouti "A simple transmit diversity technique for wireless communications", IEEE J.Select Areas Commun.,1998.

[8] 3GPP "Feasibility Study for Orthogonal Frequency Division Multiplexing (OFDM) for UTRAN enhancement" TR 25.892 V6.0.0, 2004-06.

[9] C. Spiegel, J. Berkmann, Z. Bai, T. Scholand, C. Drewes "MIMO Schemes in UTRA LTE, A comparison" IEEE, 2008

[10] K.F. Lee, D.B. Williams "A Space-Frequency Transmitter Diversity Technique for OFDM Systems" IEEE, 2000 